\title{
Impact of Low Intensity Millimeter-Waves on Cell Membrane Permeability
}

\author{
Peter H. Siegel ${ }^{\mathrm{a}}$ and Victor Pikov ${ }^{\mathrm{b}}$ \\ ${ }^{a}$ California Institute of Technology, Pasadena, CA 91125 USA \\ ${ }^{\mathrm{b}}$ Huntington Medical Research Institute, Pasadena, CA 91105 USA
}

\begin{abstract}
As the applications and commercial uses of millimeter- and submillimeter-waves grow, we should take a more detailed look at the impact this frequency range has on biological systems. This paper examines one specific effect of low-level (10-50 times the MPE - maximum permissible exposure) $60 \mathrm{GHz} \mathrm{CW} \mathrm{RF} \mathrm{power} \mathrm{on} \mathrm{cells} \mathrm{-} \mathrm{the} \mathrm{opening} \mathrm{of} \mathrm{voltage}$ sensitive cation channels.
\end{abstract}

\section{INTRODUCTION AND BACKGROUND}

$\mathrm{T}$ HE proposed widespread use of millimeter wave generators for wireless telecommunications [1] should have prompted renewed scientific interest in the effects of this wavelength range on biologic materials and organisms. Yet there is little or no funding from US Federal Agencies, and by extension, very few research groups working in this area. Several recent investigations outside the US [e.g. 2-3] have noted significant impact from millimeter wave exposures that are not that much higher than the current MPE limits of $1 \mathrm{~mW} / \mathrm{cm}^{2}$. In at least one set of experiments [4] millimeter wave heating of skate skin had the opposite effect on nerve activity as general radiant heating, causing a suppression of neuronal firing rather than an enhancement. Higher levels of power $\left(61 \mathrm{GHz}, 190 \mathrm{~mW} / \mathrm{cm}^{2}\right)$ have resulted in the appearance of cation channels in snail neuron membranes [5].

\section{EXPERIMENTS}

In our experiments we have begun to examine the impact of modest levels of RF power $\left(10-100 \mathrm{~mW} / \mathrm{cm}^{2}\right)$ on specific cell processes, with the hope that at least some of these processes can be controlled with the application of millimeter waves. As a first step to understanding where the RF energy distributes in a microscopic region in and around cells, we developed a non-contact method of monitoring the temperature of individual live cells in media. The technique uses microscopic Raman spectroscopy to monitor temperature dependent changes in the $\mathrm{O}-\mathrm{H}$ vibrational modes of water at optical wavelengths, where penetration and scattering is not a serious issue as it is in IR spectroscopy. We were able to correlate and record changes in the measured spectra of DI water, cells in RPMI (Roswell Park Memorial Institute) media and cells in PBS (phosphate buffered saline) as the temperature was changed from 25-40C. We were also able to record the temperature rise of cells in RPMI when exposed to modest levels (up to $100 \mathrm{~mW} / \mathrm{cm}^{2}$ ) of millimeter wave power for 2 minutes, and showed that these temperatures were higher than those that would be recorded by a commercial IR camera focused on the cells plus the surrounding media.
In a more recent experiment, still preliminary at this juncture, we transfected the peGFP-F vector into an immortalized line of human derived lung cells (designation H1299). The resultant fGFP (farnesylated Green Fluorescent Protein) locates on the inner cell membrane where it fluoresces green $(520 \mathrm{~nm})$ upon exposure to UV (490nm). Using oxonol as a quenching agent in the surrounding media we performed a single FRET (Förster resonance energy transfer) experiment to look for the appearance of nanoporation or cation channels in the cell membrane upon exposure to millimeter waves. If the oxonol traverses the membrane the resulting fluorescent signature shifts from green to red according to the FRET process. The figure below shows the results of the experiment, where not only did the millimeter-waves $(50 \mathrm{GHz}$ at approximately $15 \mathrm{~mW} / \mathrm{cm}^{2}$ for 2 minutes) induce ion channels to open up, but they allowed the channels to reseal upon cessation of the RF exposure after 3 minutes.

Although only a single experiment has been performed to date the data is intriguing enough to lead us to conclude that millimeter waves may indeed have more subtle effects upon biological systems than may have previously been thought. Many more experiments (and proposals) are planned.

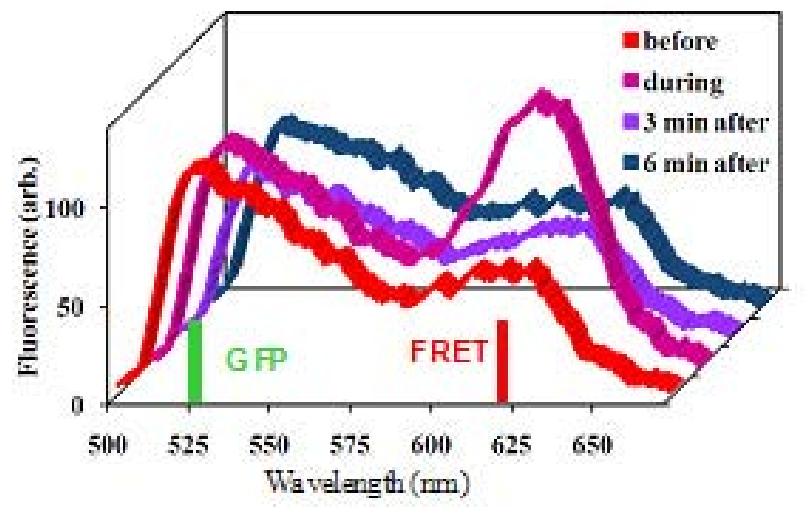

REFERENCES

[1]. FCC (2008) Millimeter Wave 70-80-90 GHz Service. In: http://wirelessfccgov/services/indexhtm?job=service home\&id=millimeter $\mathrm{w}$ ave [2]. S.M. Minasyan, G.Y. Grigoryan, S.G. Saakyan, A.A. Akhumyan, V.P. Kalantaryan, "Effects of the action of microwave-frequency electromagnetic radiation on the spike activity of neurons in the supraoptic nucleus of the hypothalamus in rats," Neurosci Behav Physiol 37:175-180, 2007.

[3]. A.G. Pakhomov, H.K. Prol, S.P. Mathur, Y. Akyel, C.B. Campbell,"Search for frequency-specific effects of millimeter-wave radiation on isolated nerve function," Bioelectromagnetics 18:324-334, 1997.

[4]. A.Y. Sazonov, V.D. Avelev, "Research of biophysical mechanisms of mm-wave effects with use of the bio-technical complex on the basis of skate electroreceptors," Millimeter Waves Med Biol 28:110-115, 2000.

[5]. S.I. Alekseev and M.C. Ziskin, "Effects of millimeter waves on ionic currents of Lymnaea neurons," Bioelectromagnetics 20:24-33, 1999. 